\title{
TEACHING UNDERGRADUATE POWER SYSTEM COURSES WITH THE USE OF SIEMENS PTI PSS/E- UNIVERSITY SIMULATION SOFTWARE
}

\author{
Chi Tang, Adam Freeman, Jerome Spence, Matthew Bradica, and Donge Ren \\ McMaster School of Engineering Technology \\ cktang@mcmaster.ca freema@mcmaster.ca spencjh@mcmaster.ca bradicm@mcmaster.ca rend@mcmaster.ca
}

\begin{abstract}
This paper summarizes the experience in using Siemens PSSE-University power system simulation software as a teaching aid to introduce basic power system concepts to engineering technology students at the undergraduate level. Topics to be discussed and demonstrated in this paper include AC and DC power flow analysis, power system transient stability analysis, reactive power and voltage control, as well as short circuit analysis.

For each of the topics presented above, the paper will provide (i) the theoretical background, (ii) a numerical example solvable by hand-calculations, and (iii) the corresponding solution obtained from running PSSEUniversity. The paper will also provide the perspectives of the instructor on using PSSE as a teaching tool and the perspectives of the students on using PSSE as a learning tool.
\end{abstract}

Keywords: Power engineering education, experiential learning, power system simulation, load flow analysis, transient stability analysis, short circuit calculation

\section{INTRODUCTION}

The province of Ontario promotes the development of renewable energy to replace its coal-fired generating plants. This policy has attracted a large number of engineering and technology students into the area of power system and energy engineering. At McMaster University, a number of power system courses are currently offered to the Bachelor of Technology (BTECH) students who have registered in the Energy Engineering Technologies Program, Faculty of Engineering. The BTECH program is now under the administration of McMaster School of Engineering Technology.

This paper summarizes the experience in using Siemens PSSE-University power system simulation software as a teaching aid to introduce basic power system concepts to engineering technology students at the undergraduate level. Topics to be discussed and demonstrated in this paper include AC and DC power flow analysis, power system transient stability analysis, reactive power and voltage control, as well as short circuit analysis.

Siemens PSSE is a major power system simulation software currently used by a majority of power system engineers in North America to analyze and assess power system steady state and dynamic performance. In Ontario, all key players in the power industry such as the System Operator, the System Planner and the Transmission Providers all use this simulation software to perform power system analysis. Therefore, it is highly beneficial to introduce the tool to all engineering and technology students at an early stage of their undergraduate education. These students now have access to the university version of PSSE free of charge at the courtesy of Siemens.

For each of the topics presented above, the paper will provide (i) the theoretical background, (ii) a numerical example solvable by hand-calculations, and (iii) the corresponding solution obtained from running PSSE University. The paper will also provide the perspectives of the instructor on using PSSE as a teaching tool and the perspective of the students on using PSSE as a learning tool.

\section{POWER SYSTEM COURSES}

At McMaster School of Engineering Technology, a number of power system courses are offered under the Energy Engineering Technology stream. They include:

i) Power Distribution I (3PD3)

ii) Power Distribution II (4PD3)

iii) Power Protection I (4PM3)

iv) Power Protection II (4PP3)

v) Renewable Energy I (4RE3)

vi) Renewable Energy II (4RT3)

vii) Power Quality (4PQ3)

viii) Power Generation (3EP3) 
These courses all have some topics that can be simulated by using PSSE-University, as illustrated in Fig. 1. For example, load flow and transient stability analyses are covered in Power Distribution I and II, while short circuit analysis is covered in Power Protection I and II. Integration of renewable energy into the power grid and their impacts on power quality can also be evaluated using PSSE.

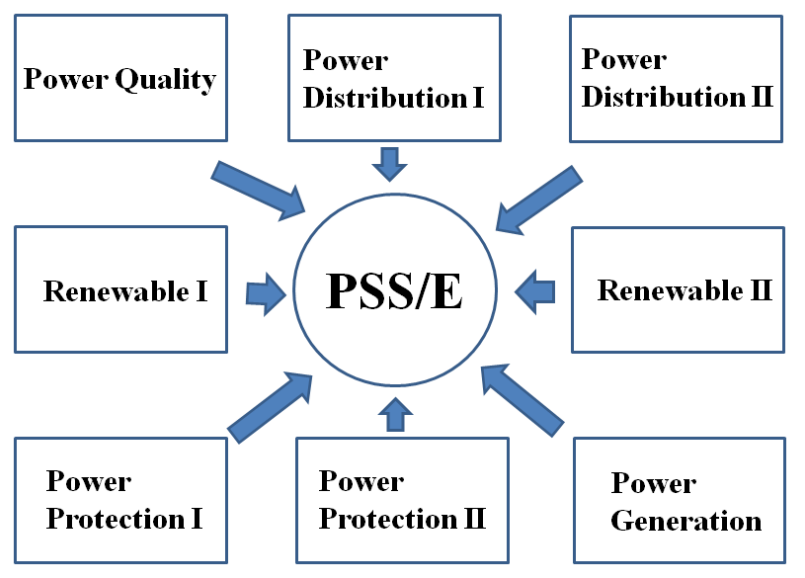

Fig. 1. Power System Courses in MSET

Summary descriptions of these courses can be obtained from the website of McMaster University.

\section{COMMERCIAL SIMULATION SOFTWARE PACKAGES}

Many commercial power system simulation software packages are available from different vendors. Listed below are some of these packages:

i) PSSE from Siemens

ii) PSLF General Electric

iii) DSA from PowerTech Labs Inc.

iv) PowerWorld from PowerWorld Corporation

v) ETAP from ETAP Canada Ltd.

vi) CYME from CYME International Inc.

In BTECH, PSSE has been chosen as a learning tool for a number of reasons. First, the Independent Electricity System Operator (IESO) and the largest transmission company HydroOne all use PSSE to evaluate transmission grid steady state and dynamic performance. As a result, transmission network models are readily available in PSSE format. In fact, PSSE is one of the main analytical tools for power engineers in the Eastern Interconnection for conducting transmission studies.

Another reason for choosing PSSE is that students can obtain a free copy of the software, PSSE-University, free of charge from Siemens. They can conduct their own simulations on their laptops without having to go to the computer lab at school, even though BTECH has purchased an educational license for PSSE. The main difference between the PSSE-University and PSSE is that the former is limited to analyzing power systems of up to 50 buses.

\section{MAJOR POWER SYSTEM CONCEPTS}

This section briefly describes three power system topics that are usually covered at the undergraduate level and demonstrate how PSSE can be used to supplement conventional classroom teaching of power system courses. The three topics are: load flow analysis, transient stability analysis, and short circuit analysis.

\subsection{Load Flow Analysis}

The main purpose of load flow analysis is to calculate bus voltages and transmission line MW and MVar flows for a given power grid. Students are exposed to two types of power flow analysis: AC power flow and DC power flow calculation.

4.1.1 AC Power Flow. A 3-bus system is shown in Figure 2 , in which buses 1 and 2 are generator buses while bus 3 is a load bus. To solve the 3-bus network, a set of algebraic equations can be formulated as follows:

$$
\begin{aligned}
& \bar{S}_{1}=\bar{V}_{1} \bar{I}_{1}^{*} \\
& \bar{S}_{2}=\bar{V}_{2} \bar{I}_{2}^{*} \\
& \bar{S}_{3}=\bar{V}_{3} \bar{I}_{3}^{*} \\
& \text { where } \\
& {\left[\begin{array}{c}
\bar{I}_{1} \\
\bar{I}_{2} \\
\bar{I}_{3}
\end{array}\right]=\left[\begin{array}{ccc}
\bar{Y}_{11} & -\bar{Y}_{12} & -\bar{Y}_{13} \\
-\bar{Y}_{21} & \bar{Y}_{22} & -\bar{Y}_{23} \\
-\bar{Y}_{31} & -\bar{Y}_{32} & \bar{Y}_{33}
\end{array}\right]\left[\begin{array}{l}
\bar{V}_{1} \\
\bar{V}_{2} \\
\bar{V}_{3}
\end{array}\right]}
\end{aligned}
$$

$\mathrm{S}_{1}, \mathrm{~S}_{2}$, and $\mathrm{S}_{3}$ are complex power injections into buses 1 , 2 , and 3 respectively. The Y-bus matrix can be formulated once the transmission elements are given. Assuming generator 1 is the slack bus, the known and unknown quantities for each bus are given in Table 1 .

Table 1. Load Flow Known and Unknown Quantities

\begin{tabular}{|l|l|l|}
\hline & $\begin{array}{l}\text { Known } \\
\text { Quantities }\end{array}$ & $\begin{array}{l}\text { Unknown } \\
\text { Quantities }\end{array}$ \\
\hline Bus 1 & $\left|\mathrm{V}_{1}\right|, \Theta_{1}$ & $\mathrm{P}_{1}, \mathrm{Q}_{1}$ \\
\hline Bus 2 & $\mathrm{P}_{2},\left|\mathrm{~V}_{2}\right|$ & $\mathrm{Q}_{2}, \Theta_{2}$ \\
\hline Bus 3 & $\mathrm{P}_{3}, \mathrm{Q}_{3}$ & $\left|\mathrm{~V}_{3}\right|, \Theta_{3}$ \\
\hline
\end{tabular}




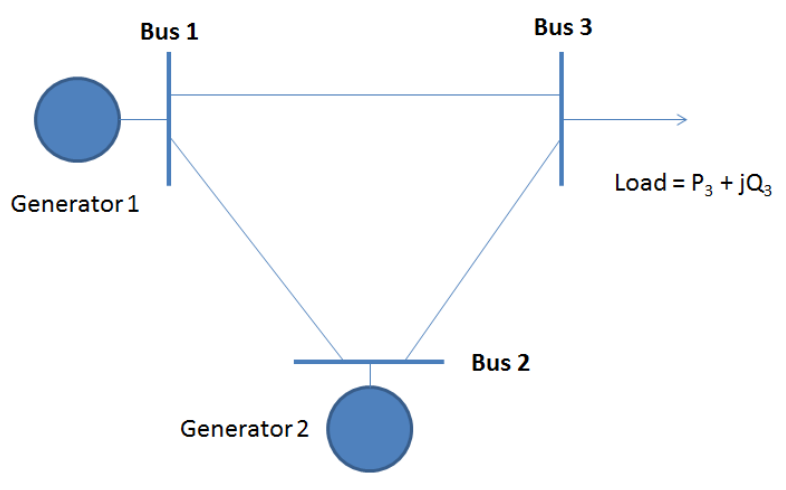

Fig. 2. 3-Bus System

Students are introduced the numerical techniques for solving AC load flow equations, such as the Gauss Seidel and Newton's iterative methods. In addition, the important concepts of stopping criteria based on power mismatch and $\Delta \mathrm{V}$ are emphasized. By solving the $\mathrm{AC}$ power flow problem, students can identify the effectiveness of various voltage control measures.

After learning the basic load flow concepts, students will have an opportunity to work on a project to determine the maximum power transfer over the Ontario north-south transmission corridor as shown in Fig. 3, following the pre- and post-contingency voltage criteria as stipulated in [3]. In doing this project, students will compare the maximum power transfer capability with and without series compensation.

4.1.2 DC Power Flow. Once the students have grasped the basic concepts of $\mathrm{AC}$ power flow, they can proceed to simplify the calculations by making certain assumptions, hence resulting in what is known as the DC load flow analysis. Given below is the formulation of DC load flow:

$$
\begin{aligned}
& -[\mathbf{B}][\boldsymbol{\theta}]=[\mathbf{P}] \\
& -[\mathbf{B}][\mathbf{V}]=[\mathbf{Q}] \\
& P_{i j}=\frac{\theta_{l}-\theta_{j}}{X_{i j}} \\
& Q_{i j}=\frac{V_{i}-V_{j}}{X_{i j}}
\end{aligned}
$$

The DC load flow equations as described above can be solved by linear techniques. PSSE offers DC power flow options and students can use it to perform fast contingency analysis to identify potential thermal overloads on the system. They can also compare the accuracy between the $\mathrm{AC}$ and DC load flow calculations.

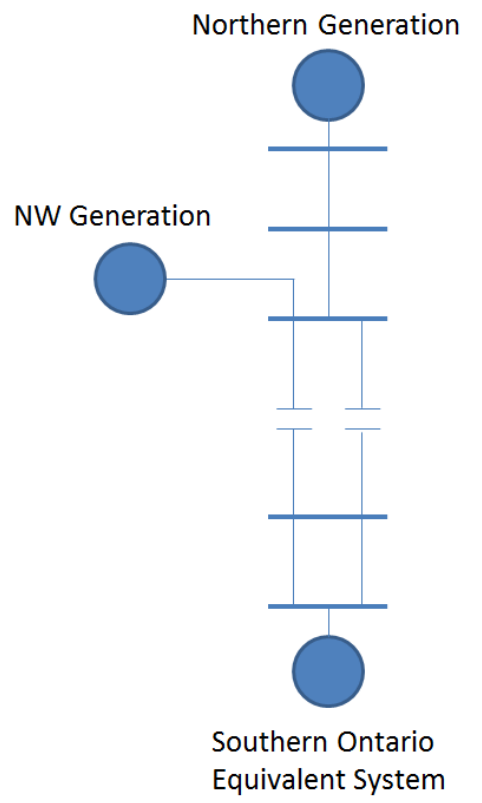

Fig. 3. Ontario North-South Transmission Corridor

4.1.3 Voltage Stability Analysis. In addition to solving for bus voltages and line flows, PSSE can also be used to derive PV and VQ curves for different contingency scenarios, a process commonly used to assess power grid voltage stability. In class, students are given a multi-bus system to learn the voltage stability phenomenon. The students through PV and VQ analysis can determine maximum power transfer capability of a transmission path while preserving voltage stability of the system. PSSE produces PV curves for each monitored bus so that the weakest node of the system can be identified. Figure 4 presents is a typical output of a PV analysis from PSSE for different contingency scenarios.

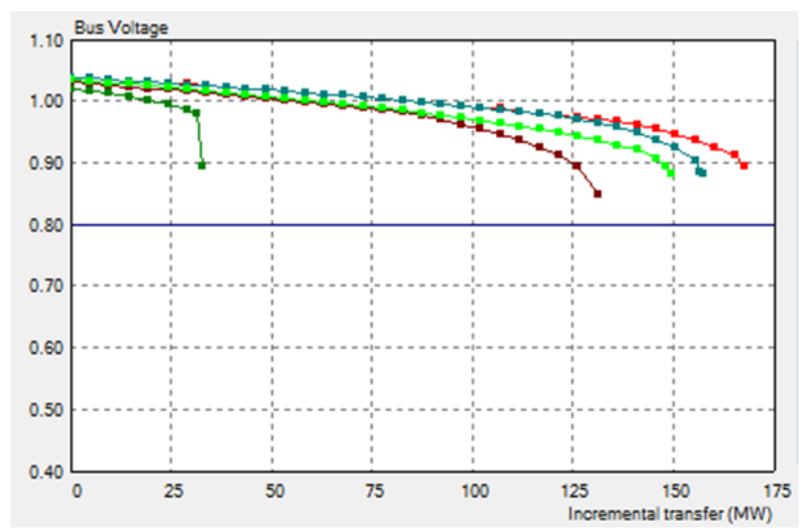

Fig. 4. PV Curve 


\subsection{Transient Stability Analysis}

Transient stability, also known as large signal stability, is a measure of the ability of synchronous generators to remain in synchronism when the power system suffers from a disturbance. The disturbance could be a fault successfully cleared by protective relays, tripping of a major generator, or loss of a large load. These types of disturbances can cause rotors to accelerate, resulting in an increase in internal angle and the potential loss of transient stability.

For a single-machine-infinite-bus system (SMIB), the electrical power output of the generator is significantly reduced during the fault-on period, so that the mechanical input of the generator exceeds its electrical output. As a result, the transient kinetic energy of the generator increases rapidly as the rotor accelerates. When the fault is finally cleared, the generator needs to dissipate all the excess transient kinetic energy into the power system. If proper protection schemes are not in place to isolate the fault fast enough, the generator can lose synchronization (i.e. falling out of step) and suffer severe damages. Transient stability is affected by many factors such as the mechanical inertia of the generator, pre-contingency generator output level, and the excitation control of the generator.

The Equal Area Criterion can be used to determine the transient stability of a SMIB if the synchronous machine is represented as a classical generator, i.e., a constant voltage source behind a transient reactance. The Equal Area Criterion states that for a given contingency, the area corresponding to the destabilizing effect of the fault must not exceed the area corresponding to the restorative strength of the post-fault system. The critical clearing time is defined when the fault is cleared so that the generator is just marginally stable. The generator angle at the critical clearing time is known as the critical clearing angle $\left(\delta_{C}\right)$.

This phenomenon is illustrated in Figure 5. Area $A_{1}$ represents the destabilizing area due to fault inception, while area $A_{2}$ represents the restorative strength of the post-fault system. The destabilizing area must be less than the stabilizing area for the system to be transiently stable. The amount of transient kinetic energy produced during a fault increases rapidly, and therefore it is important that the fault be cleared quickly so that $\mathrm{A}_{1}$ does not exceed $\mathrm{A}_{2}$.

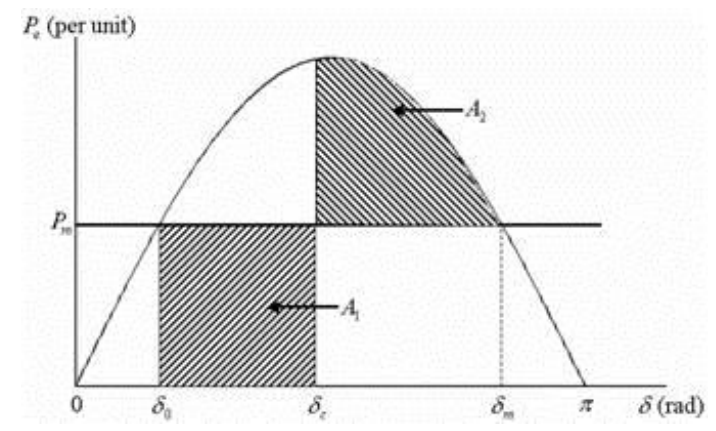

Fig. x. Critical Clearing Angle for Bolted Fault at the Generator Terminal

The Equal Area Criterion is, however, only applicable for a single-machine-infinite-bus system. When there are multiple generators, the time domain simulation technique must be used. This is the method which is used by PSSE. For the classical SMIB system, the swing curve can be computed by the numerical integration of the swing equations which are given below:

Swing Equation:

$$
\begin{aligned}
& \Delta \omega=\frac{1}{2 H} \int\left(P_{m}-P_{\theta}\right) d t \\
& \Delta \delta=2 \pi 60 \int \Delta \omega d t
\end{aligned}
$$

$\mathrm{Pm}=$ input mechanical power $[\mathrm{pu}]$

$\mathrm{Pe}=$ electrical power output $[\mathrm{pu}]$

$\mathrm{H}=$ inertia constant $[\mathrm{s}]$

$\Delta \omega=$ speed deviation $[\mathrm{pu}]$

$\Delta \delta=$ angle deviation [radians]

The interactive structure of PSSE guides students through a step-by-step process which provides a greater understanding of the major factors which influence the transient stability of a system. The student specifies the sequence of switching activities and PSSE will compute the swing curves of the monitored generators. The stability of the system for the given disturbance can be determined by examining the swing curves. Figure 6 represents a stable case in which the fault was cleared before the critical clearing time. The plot also shows that the post-fault system exhibits good damping when detailed excitation controls are represented. Figure 7 represents a system in which the fault was not cleared fast enough and the generator suffered transiently instability. From this plot it can be seen that the generator go out of step very quickly. 


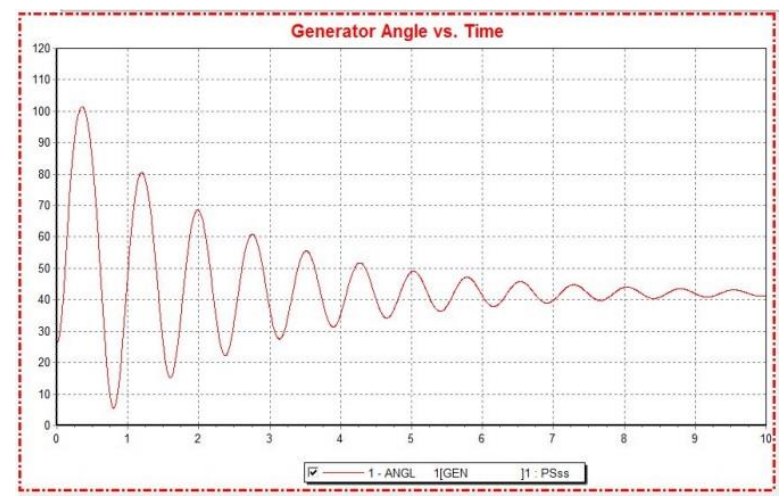

Fig. 6. Stable and Dampened Case

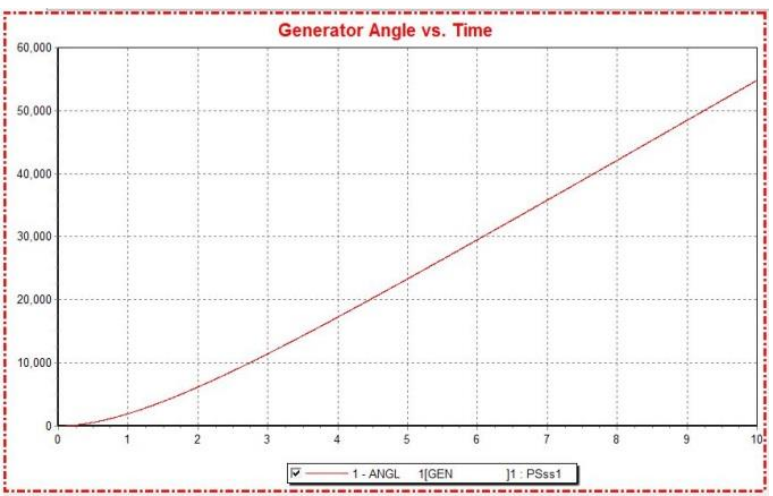

Fig. 7. Unstable Case

PSSE exposes students to analysis that cannot practically be done by hand calculations. Furthermore, the interactive nature of PSSE is conducive to sensitivity analysis, that is, different elements or variables of the system can be changed and the student can immediately see how these changes affect system stability (e.g. varying the length or location of a fault).

\subsection{Short Circuit Calculation}

In teaching power system protection, it is important to introduce fault calculation which is also known as short circuit calculation. Students are first introduced the concept of symmetrical components and the formation of positive, negative, and zero sequence networks. Then they learn how to interconnect the sequence networks depending on the type of fault under consideration, in order to calculate the fault current levels.

A simple network is shown in Figure 8 with two parallel lines between buses A and B. Assuming all network impedances are given, including the zero sequence mutual coupling between the two parallel lines, students can evaluate the fault current for a single-line-toground fault at bus B. The proper connection of the sequence networks is shown in Figure 9.

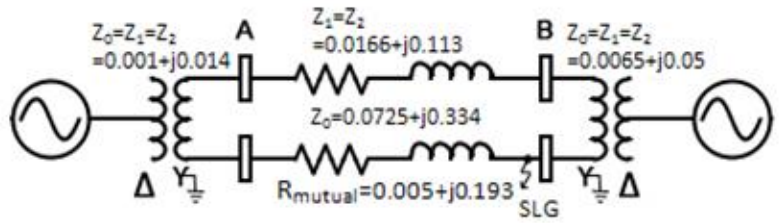

Fig. x. Simple Transmission Network

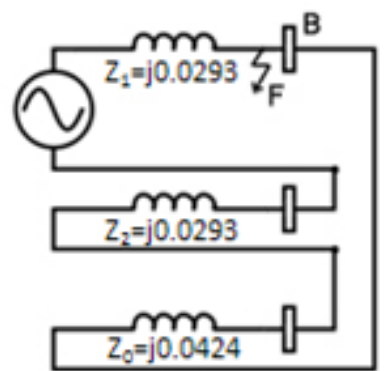

Fig. x. Connection of Sequence Networks

Hand calculations yield the following results:

SLG fault current:

$I_{a}^{f}=\frac{3 E_{a}}{z_{1}+z_{2}+z_{0}}=\frac{3 \times 1.0 \angle 0^{\circ}}{j 0.101}=29.7 \angle-90^{\circ} \mathrm{pu}$

$3 \varphi$ fault current: $I_{\alpha}^{f}=\frac{E_{a}}{z_{1}}=\frac{1.0 \angle 0^{\circ}}{j 0.0293}=34 \angle-90^{\circ} p u$

After learning the basic short circuit calculation, students can go to PSSE and repeat the calculation as well as comparing the results. Solutions obtained from PSSE are provided below:

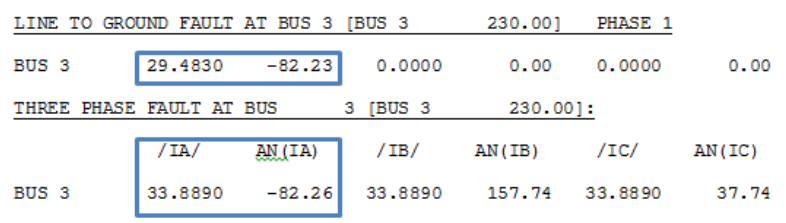

The SLG fault current is approximately $29.5 \mathrm{pu}$, compared to 29.7 pu obtained from hand-calculation. The $3 \varphi$ fault current output is 33.9 pu compared to 34 pu as obtained from hand-calculation.

Once the students are confident with the calculation procedure using PSSE, they can analyze more complex networks such as incorporation of new transmission and/or generation elements. 


\section{EXPERIENCE TO-DATE}

PSSE or PSSE-University has been introduced to BTECH students at McMaster University for about two years. This section summarizes the assessment of PSSE as a teaching tool as viewed by the instructor, and as a learning tool as viewed by the students who have used PSSE in doing course assignments or projects.

\subsection{PERSPECTIVE OF INSTRUCTOR}

Modern power systems are complex and full of advanced control devices such as Flexible AC Transmission Systems (FACTS) devices with an increasing penetration of renewable energy resources. Traditional ways of teaching undergraduate power system courses based on a simple single-machine-infinite-bus system can be augmented by using commercial grade power system simulation software such as PSSE. In doing so, power engineering and technology students no longer spend a lot of time solving manually algebraic and/or differential equations governing the power system steady state and dynamic behavior. Instead, they can devote more time to familiarizing with the characteristics of the real transmission grid and investigating the real power system operating issues such transient and/or voltage instability arising from insufficient transmission capacity.

\subsection{PERSPECTIVE OF STUDENTS}

PSSE allows students to see the type of computational power that is used in the industry for complex multi-bus systems. Students find this beneficial in addition to hand calculations because it is a way to confirm calculations that have been worked on paper. It also gives the students the ability to change system parameters and see the "what if" scenarios on small power systems. Being able to do sensitivity analysis is a great way to learn different power flow concepts.

Some students are visual learners. Being able to do analysis such as PV and VQ analysis as well as dynamic simulation appeals to their learning style and has helped them with the retention of the various topics learned. Doing assignments where analysis of small systems is required has also brought more meaning to the theory and they are able to anchor it with a practical application.

\section{CONCLUSION}

At McMaster School of Engineering Technology, PSSE-University has been used by their Energy Engineering Technology students for about two years to learn major power engineering concepts such as load flow, transient stability, and short circuit analysis. Experience to date has indicated that using a commercial power system simulation software can help students master major power system concepts much faster. In addition, they can also get a chance to learn the real power system based on network models provided by the power industry.

\section{References}

[1] Siemens PTI PSSE 33.2 Program Application Guide, Volumes I and II, Revised July, 2012, Siemens Industry, Inc., Siemens Power Technologies International

[2] Siemens PTI PSSE 33.2 Program Operation Manual, Revised July, 2012, Siemens Industry, Inc., Siemens Power Technologies International

[3] Ontario Resource and Transmission Assessment Criteria, Issue 5.0, The Independent Electricity System Operator, Revised August 22, 2007 